\title{
Soria 3: nuevas evidencias de la ocupación aldeana temprana en Yocavil, Noroeste argentino. Un ejercicio interpretativo
}

\author{
Alina Álvarez Larrain, Romina Spano y María Solange Grimoldi \\ IDECU-CONICET, Museo Etnográfico Juan B. Ambrosetti \\ Facultad de Filosofía y Letras, Universidad de Buenos Aires \\ alinaalvarezlarrain@gmail.com romina.spano@gmail.com solgrimoldi@gmail.com
}

Recibido: $29 / 8 / 2015$

Aceptado: 23/5/2016

\begin{abstract}
RESUMEN
Se presentan evidencias estratigráficas provenientes de un sondeo localizado en el interior de una estructura semicircular con muros de piedra, sitio denominado Soria 3, correspondiente al período Temprano (600 a.C.-1000 d.C.) del Noroeste argentino. La unidad, registrada en la Mesada de Andalhuala Banda (Valle de Yocavil, Provincia de Catamarca), se interpretó como una unidad residencial empleada, luego de su desocupación, como espacio funerario para el entierro de un infante en una olla. Se detallan las características constructivas de la estructura, la estratigrafía y los materiales recuperados, la modalidad de inhumación, el contenedor cerámico empleado para la misma y los resultados del análisis bioarqueológico de los restos óseos. Asimismo, se comunican las dataciones radiocarbónicas obtenidas a partir de muestras del individuo y carbones del piso de ocupación del espacio doméstico. Las evidencias son coherentes con el patrón de ocupación previamente registrado en el sitio Soria 2, unidad doméstica emplazada en la misma mesada, apuntando a la configuración de un patrón aldeano local.
\end{abstract}

Palabras clave: Período Temprano o Formativo, Noroeste argentino, valle de Yocavil, unidad doméstica, entierro de infante en olla.

\section{Soria 3: New Evidences of Early Village Occupation in Yocavil, Northwest Argentina. An Interpretative Exercise}

\begin{abstract}
Stratigraphic evidence from a test excavation located inside a semicircular structure with stone walls, called Soria 3 from the Early Period (600 B.C.-A.D. 1000) is presented. The unit, registered in the Mesada of Andalhuala Banda (Yocavil Valley, Northwest Argentine), was interpreted as a residential unit that was used after its abandonment as a funerary space for the burial of an infant within a ceramic vessel. The construction features of the structure, the stratigraphy and materials recovered, the mode of burial, the ceramic vessel, and the results of bioarchaeological analysis are described. Radiocarbon dates obtained from samples of the individual and charcoal of an occupation floor corresponding to a domestic space are also reported. The evidence is consistent with the pattern previously recorded in Soria 2 located in the same piedmont glacis, pointing to the configuration of a local village pattern.
\end{abstract}

Key words: Early or Formative Period, Northwest Argentine, Yocavil Valley, domestic unit, infant pot burial.

Sumario: 1. Introducción. 2. La Mesada de Andalhuala Banda. 3. Soria 3-UA 11. 4. Análisis de Soria 3-UA 11: un ejercicio interpretativo. 5. Materialidad temprana y ritual fúnebre, I: las ollas ordinarias como urnas funerarias. 6. Materialidad temprana y ritual fúnebre, II: las casas como tumbas. 7. Comentarios finales. 8. Referencias bibliográficas.

\section{Introducción}

Las dificultades para reconstruir el mundo agroalfarero temprano en Yocavil (Noroeste argentino) han sido tratadas en otros trabajos (v. g. Palamarczuk et al. 2007; Scattolin 2000; Tarragó y Scattolin 1999). En comparación con la alta cantidad de sitios arqueo- 
lógicos del período Tardío (1000-1480 d.C.) registrados en el valle de Yocavil, las evidencias de lugares correspondientes al período Temprano o Formativo (200 a.C.-1000 d.C., sensu Núñez 1978: 468) se reducen aún a pocos sitios (véase Scattolin 2007 y Tarragó y Scattolin 1999 para una síntesis). Entre otras razones, la persistente ocupación del valle a lo largo de los últimos mil años ha generado una suerte de «palimpsesto acumulativo» y «multi-temporal» (Bailey 2007: 204; Olivier 1999: 127), en el cual se superponen los restos materiales de distintas dinámicas sociales del pasado. Estos paisajes multi-temporales se han registrado, por ejemplo, en la zona de Caspinchango (Álvarez y Lanzelotti 2013), en el sector tucumano de la Sierra de Quilmes (Maldonado et al. 2012) y en Andalhuala Banda, como veremos aquí.

La Mesada de Andalhuala Banda (en adelante MAB), constituye un caso paradigmático de la dificultad de encontrar ocupaciones tempranas en el sur del valle de Yocavil, dada la ausencia en superficie de estructuras arquitectónicas habitacionales asignables a alguno de los patrones de asentamiento conocidos para tiempos tempranos en el área -como por ejemplo el denominado «Tafí», entre otros (González y Núñez 1960; Scattolin 2010)-, y por la prolongada ocupación de este espacio que conllevó remodelaciones constructivas. En el marco de la investigación doctoral de una de las autoras, se llevó a cabo el levantamiento sistemático de la arquitectura de superficie de la mesada y se realizaron excavaciones exploratorias con el objetivo de obtener materiales en capa útiles para estimar la cronología de los espacios habitados (v. g. restos orgánicos datables por el método de radiocarbono o cerámica decorada diagnóstica) (Álvarez 2015). El muestreo realizado para los sondeos incluyó una unidad arquitectónica, a la cual actualmente denominamos sitio Soria 3-UA 11, cuya modalidad constructiva sugería su adscripción a tiempos tempranos. No obstante lo acotado de la superficie de excavación $\left(1 \mathrm{~m}^{2}\right)$, el hallazgo efectuado y su contexto nos llevaron a detenernos en él y practicar un ejercicio interpretativo sobre sus implicaciones para la problemática planteada líneas arriba. El hallazgo de un entierro de neonato dentro de una olla ordinaria permitió no sólo obtener dataciones tempranas de máxima certeza (nuestro objetivo inicial), sino también tratar el tema de la configuración de un patrón aldeano y la manipulación de cuerpos de neonatos en el marco comunitario del mundo formativo.

\section{La Mesada de Andalhuala Banda}

La MAB constituye un glacis de origen cuaternario localizado entre el fondo del valle de Yocavil y la Sierra del Aconquija (Figura 1). Con una superficie aproximada de 186 ha, se ubica entre las cotas de 2.100 y $2.300 \mathrm{~m} \mathrm{s.n.m}$., con una pendiente general de $5,18 \%$ que desciende hacia el noroeste.

Trabajos emprendidos una década atrás permitieron identificar la presencia de terrazas y unidades habitacionales dispersas. Bajo la presunción de que se trataba de un área de producción agrícola, se encaró en el año 2002 un sondeo exploratorio en un ángulo conformado por dos líneas de piedras, con el objetivo de obtener muestras para realizar una datación radiocarbónica que sirviera como una primera referencia para establecer la cronología de este espacio. El sondeo permitió dar con una estruc- 
tura de piedra cuyo trazado era apenas observable en superficie (Palamarczuk et al. 2007: 124). Las excavaciones en área realizadas posteriormente revelaron que se trataba de los restos de una estructura habitacional compuesta por, al menos, dos recintos subcuadrangulares adosados, a la cual se denominó sitio Soria 2; una datación convencional de carbón de un fogón asociado al piso de ocupación arrojó una edad de 1940 \pm 80 AP (LP-1541), con un rango de edad calibrada de 53 a.C.-342 d.C. (Palamarczuk et al. 2007: 127). Se trata de una vivienda del Formativo empleada, tras su abandono, como área funeraria: se encontraron tres inhumaciones que incluyen un entierro primario de neonato en urna dentro de un foso, un entierro primario de neonato en urna dentro de un compartimento de piedra y un entierro secundario contenido en otro compartimento de piedra que involucró los restos de al menos dos in-

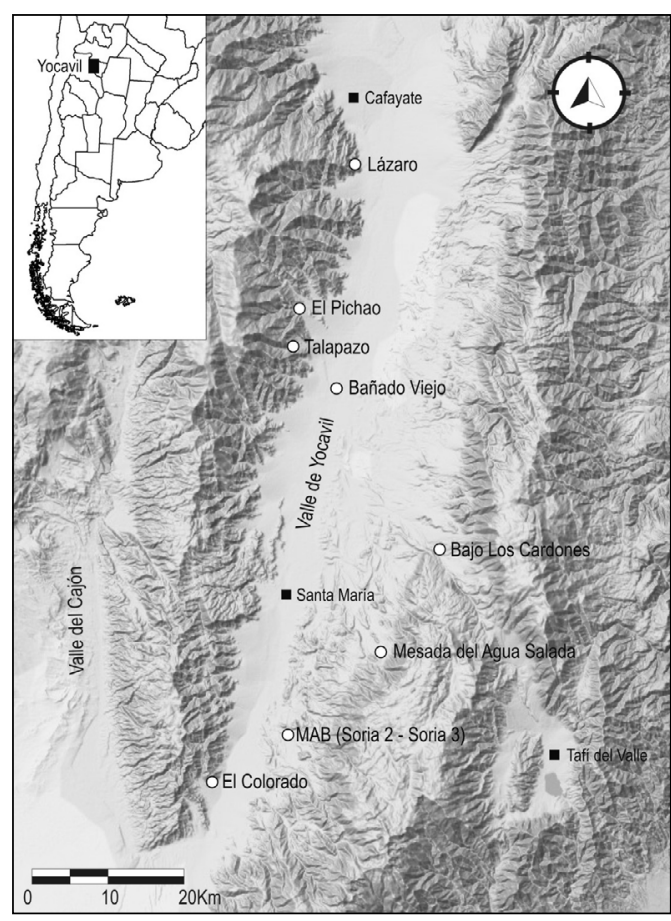

Figura 1: Mapa del valle de Yocavil con la ubicación de algunos sitios tempranos conocidos. dividuos (un niño de alrededor de tres años de edad y un feto a término o neonato). La antigüedad de los restos, estimada mediante la datación por AMS de muestras de los mismos, se encuentra comprendida entre $1766 \pm 46$ AP (AA-87353) y $1730 \pm 46$ AP (AA-87351); estas dataciones abarcan un rango de edad calibrada que se inicia entre los años 176 al 433 d.C. y que termina entre los años 244 al 532 d.C. (Spano et al. 2014: 152, 160). El tiempo de uso del espacio como vivienda y su tiempo de empleo como lugar funerario se encuentran comprendidos dentro del período Temprano, aunque pudo haber transcurrido algún tiempo, no demasiado prolongado, entre el abandono de la casa y la ejecución de los entierros (Spano et al. 2014).

La investigación desarrollada en Soria 2 resultó fructífera al permitirnos estudiar un contexto primario con dataciones seguras que constituyó una «ventana» para asomarnos al mundo de las sociedades agropastoriles tempranas vallistas. Asimismo, nos posibilitó acceder a uno de los pocos casos conocidos localmente de entierros tempranos de infantes en vasijas. Ahora bien, esta mirada en detalle del microcosmos social que constituyó Soria 2 no resolvía, por sí sola, cuestiones referidas al uso de la MAB como espacio de residencia comunitario en tiempos tempranos.

Nuevos levantamientos arquitectónicos emprendidos en el glacis a partir del año 2010 permitieron registrar 382 unidades arquitectónicas (en adelante UA) entre las cuales se observaron habitaciones, recintos y terrazas de siembra, montículos, implementos de molienda (fijos y móviles) y posibles depósitos de almacenaje, entre otros 
(Álvarez 2015). A partir de una primera observación de la ubicación de las distintas UA en el glacis, notamos que la distribución de construcciones no era homogénea en términos cuantitativos y tipológicos. El sector con mayor densidad constructiva era el este del glacis, área que se presentó justamente como la más compleja de registrar. Allí se observaron estructuras parcialmente enterradas o modificadas y superposiciones que complican el entendimiento de las plantas arquitectónicas e indican un prolongado uso y remodelación de la arquitectura.

Las tareas de levantamiento posibilitaron, no obstante, distinguir dos modalidades de uso del glacis. La mayoría de las UA responde a tipos productivos (terrazas y recintos de siembra, depósitos y morteros fijos), con un bajo número de recintos habitacionales dispersos entre ellos. Un alto porcentaje de estas habitaciones presenta arquitectura de muros dobles con relleno -modalidad típica del Tardío de Yocavil-, bien conservadas, con abundante material cerámico en superficie de los estilos tardíos San José/Shiquimil, Loma Rica Bicolor y Santamariano (Greco 2012). La realización de sondeos en dos UA permitió obtener muestras para fechar cuyas dataciones confirman la cronología estimada a partir de la evidencia arquitectónica y artefactual de superficie (Álvarez 2015). La mesada se interpreta entonces, por un lado, como una instalación productiva dentro del sistema de asentamiento regional tardío, donde se ven reflejadas distintas instancias del ciclo productivo agrícola, sector posiblemente ligado al poblado de la Loma Rica de Shiquimil que se ubica al norte de la mesada.

Por otro lado, se registró una segunda modalidad de uso del espacio en el sector oriental, relacionada con períodos previos. Al menos el $12 \%$ del total de las unidades habitacionales observadas en la MAB correspondió a conjuntos compuestos exclusivamente por recintos circulares o subcirculares, patrón desconocido en tiempos tardíos; por esta razón se conjeturó que los mismos podían pertenecer a momentos tempranos. Considerando conjuntamente la distribución de cerámica de estilos tempranos (Plomiza pulida, Ordinaria alisada, Ante Pulido, Guachipas, entre otros) (Spano 2011) y la presencia de las UA con muros de morfología y tipo de planta semejantes a Soria 2, se propuso una superficie mínima de 35 ha de ocupación temprana en el este del glacis (Álvarez 2015). Notamos que la fisonomía aldeana temprana había sido sustancialmente alterada por procesos de sedimentación y remodelaciones posteriores del espacio para nuevos usos. Con el objetivo de obtener materiales en capa como única vía conducente a la determinación cronológica de la ocupación temprana, se seleccionó como área a sondear la UA 11, ubicada en el sector oriental del glacis y a $300 \mathrm{~m}$ al noroeste de Soria 2 (Figura 2).

\section{Soria 3-UA 11}

La UA 11 está compuesta por un recinto semicircular de $4 \times 7 \mathrm{~m}$ de planta interna. Sus muros son dobles sin relleno, de grandes mampuestos acomodados, de 0,50 a $0,80 \mathrm{~m}$ de ancho (Figura 3). Es posible que esta unidad constara de un segundo recinto, del cual sólo es visible un muro adosado al Recinto 1. Asociadas a éste se observaron líneas de muros, simples y dobles, cuya disposición no pertenece a la planta original de la estructura. 


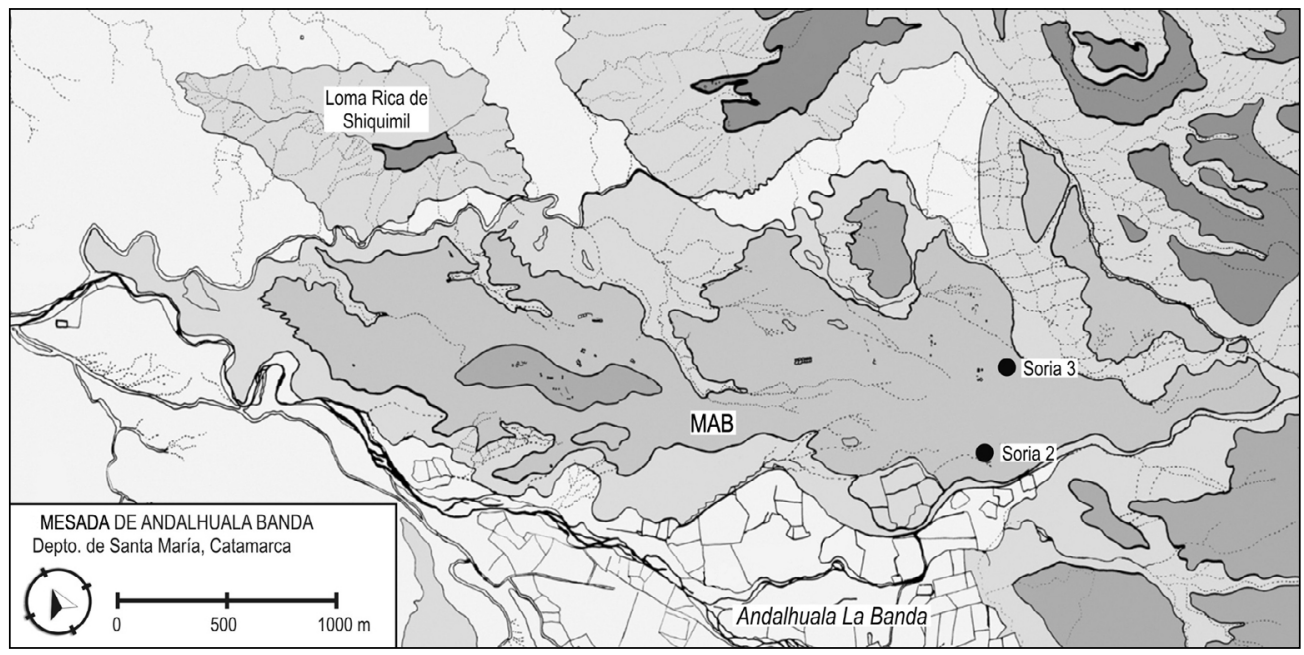

Figura 2: Mapa de la Mesada de Andalhuala Banda con la ubicación de Soria 2 y Soria 3.

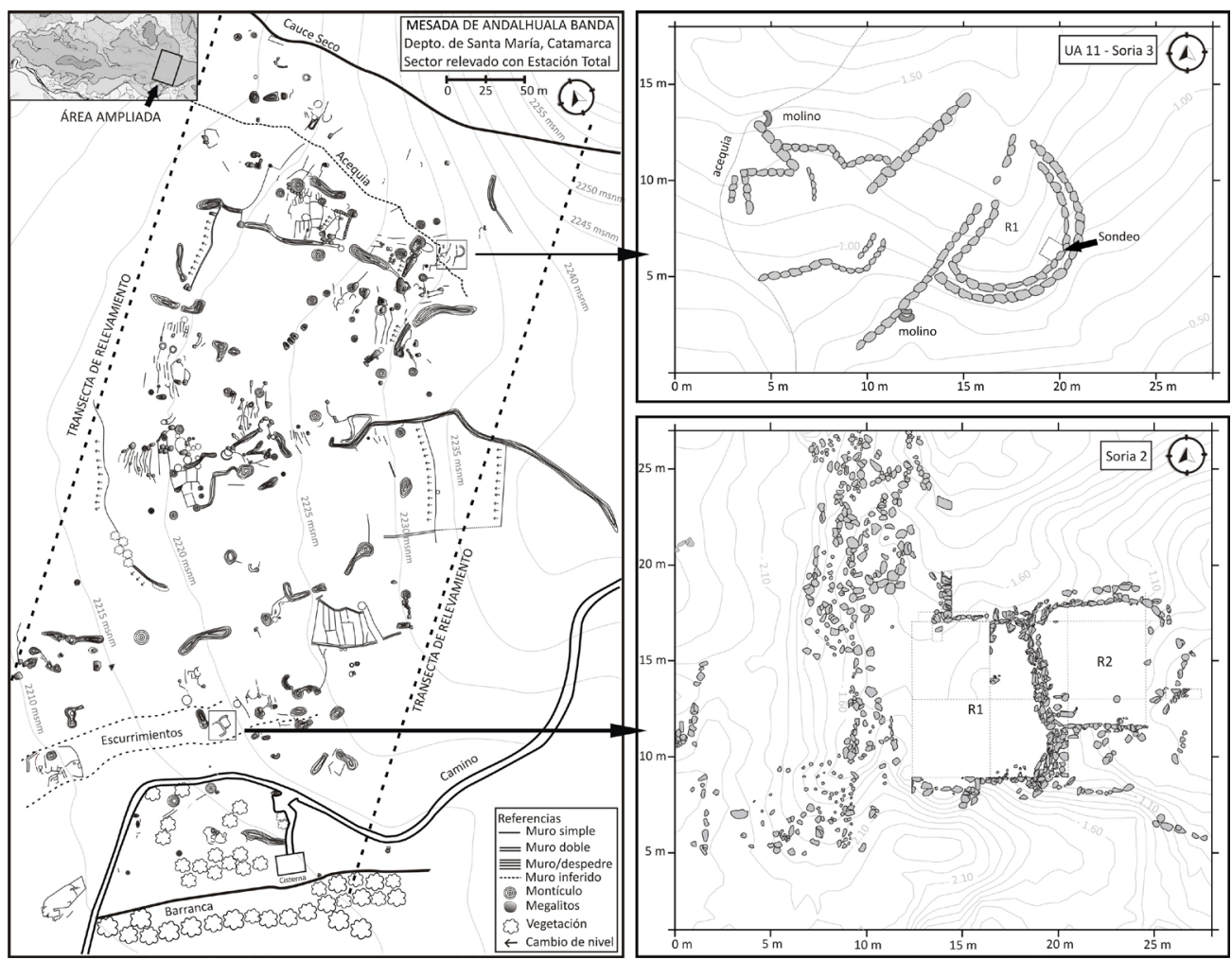

Figura 3: Transecta de levantamiento con estación total de la MAB (izquierda) y planimetrías de Soria 2 y Soria 3 (derecha). (Las curvas de nivel están expresadas en metros con respecto a la altura del datum). 


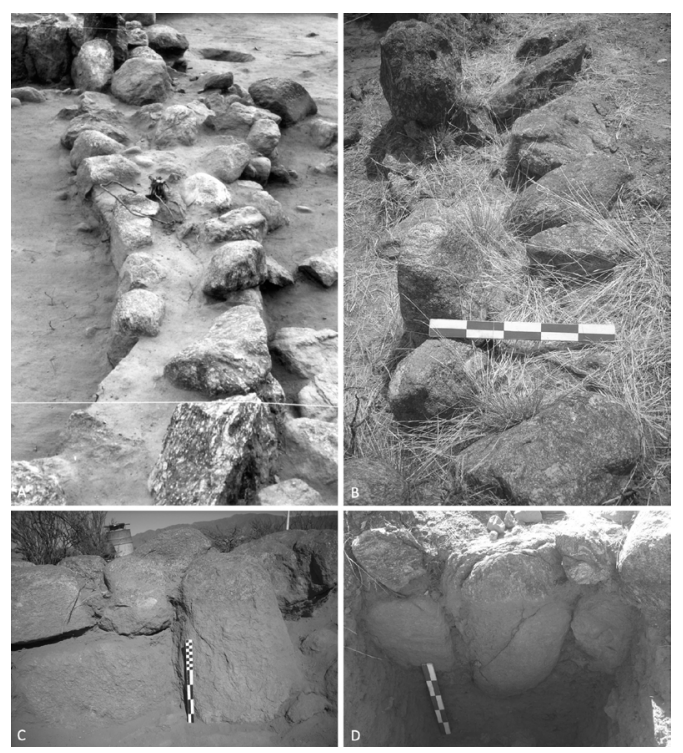

Figura 4: Muros de piedra tempranos: (a) y

(c) vista superior y de paño interno del muro que une el R1 y el R2 de Soria 2; (b) y (d) Vista superior y de paño interno del muro curvo de la UA 11.
La consideración de la morfología de los muros y el tipo de mampuestos, semejantes a los registrados en Soria 2, fue el principal criterio considerado para su excavación, en la creencia de que se trataba de una unidad temprana (Figura 4). Además, en la superficie interna del recinto se recuperó un fragmento de cerámica ordinaria con aplique en pastillaje y un fragmento de alfarería fina con grabado post-cocción, ambos similares a materiales obtenidos en Soria 2.

\subsection{Excavación y estratigrafía}

La cuadrícula de excavación se ubicó en el interior del recinto, contra el muro curvo. El sondeo alcanzó una profundidad de casi $0,90 \mathrm{~m}$, excavándose 12 niveles artificiales más una caja de 0,40 m de lado para confirmar la presencia del basamento pétreo natural de la mesada (Figura 5). Uno de

los mampuestos del muro observado en superficie se continuaba $0,60 \mathrm{~m}$ por debajo del nivel actual (ver Figura 4d). En sus laterales presentaba mampuestos más pequeños, acomodados con sus caras planas mirando hacia el interior.

El estrato de relleno, con una matriz limo-areno-arcillosa, alcanzó una potencia promedio de $38 \mathrm{~cm}$; el mismo contenía abundante material cerámico temprano, restos óseos y carbón disperso. Por debajo se definió un estrato de transición hacia el piso de ocupación de la unidad, de casi $6 \mathrm{~cm}$ de potencia, con un sedimento más suelto, del que se recuperó medio molino, un fragmento grande de cerámica ordinaria, un núcleo de andesita, huesos de camélido y varias piedras. Estos hallazgos parecen responder a la remoción de materiales de los niveles inferiores y al derrumbe de pequeñas piedras del muro. Por debajo de este estrato se registró un sedimento mucho más compacto, de litología homogénea, en el que se recuperó cerámica temprana, restos líticos, restos óseos, carbones dispersos, semillas quemadas y una concreción de pigmento blanco con marcas de manipulación; estos elementos, considerados en conjunto, nos llevan a interpretar esta capa, de una potencia acumulada de unos $20 \mathrm{~cm}$, como el piso de ocupación de la vivienda. Atravesando este estrato y en el centro de la cuadrícula se registró una olla ordinaria que se encontraba colmatada de sedimento y contenía restos humanos (véase el análisis bioarqueológico más abajo). Por debajo del piso, se registró un sedimento con ripio suelto, acompañado por una reducción notable de la frecuencia de hallazgos, de unos $10 \mathrm{~cm}$ de potencia, interpretado como la transición hacia el estrato estéril. Este último fue definido a partir de una continuidad en el sedimento con ripio suelto y de la escasez y reducido tamaño del material cultural. 


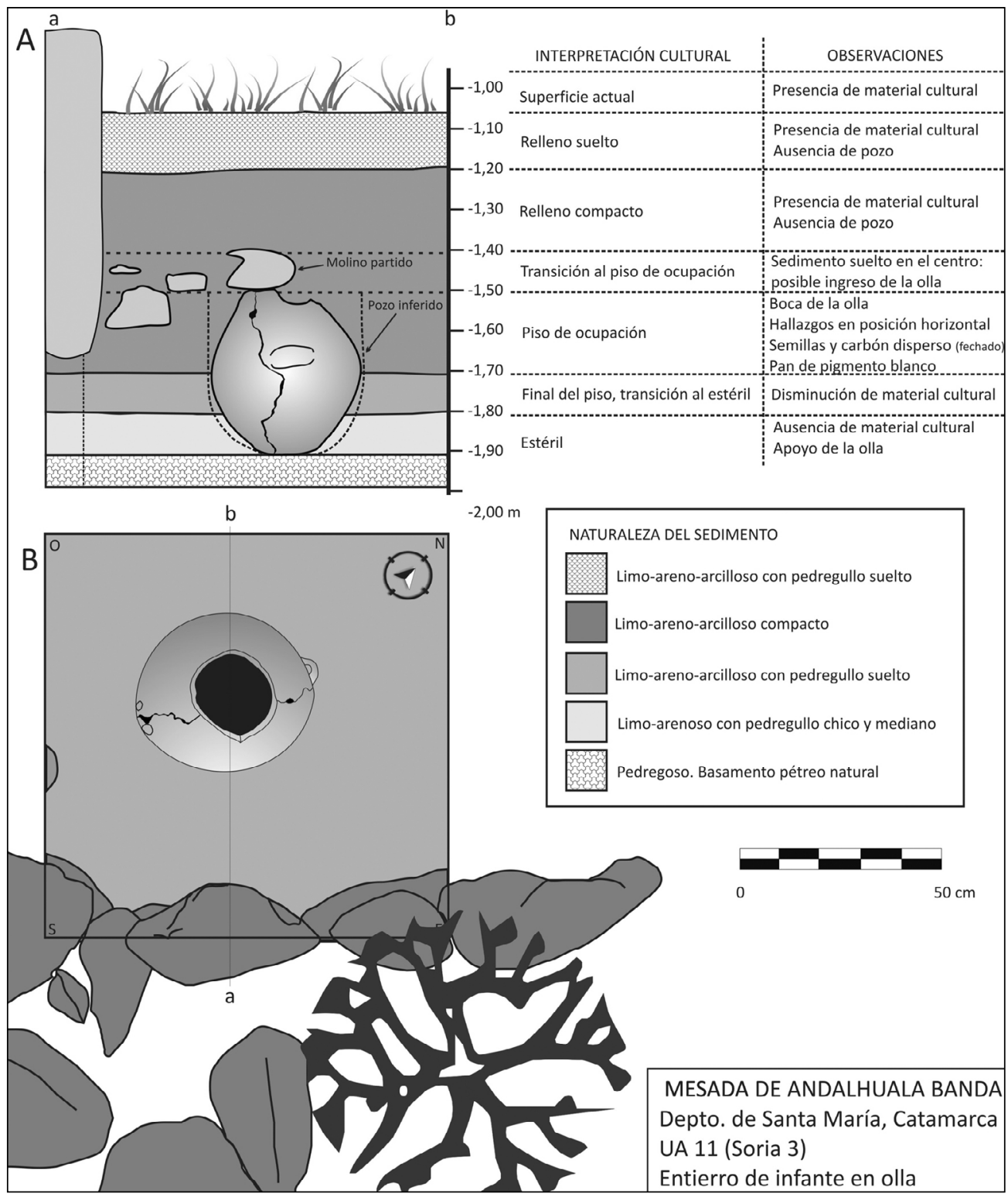

Figura 5: Sondeo de la UA 11: (a) corte de perfil a-b con reconstrucción del entierro; (b) planta de excavación del nivel 10 donde se observa el diámetro mayor de la vasija.

\subsection{Contenedor cerámico y modalidad de entierro}

La olla que funcionaba como contenedor funerario es de tipo ordinario, de forma subesferoidal restringida, borde evertido y boca estrecha. Presenta dos asas horizontales de doble inserción por remache y sección oval, ubicadas de manera asimétrica cerca 

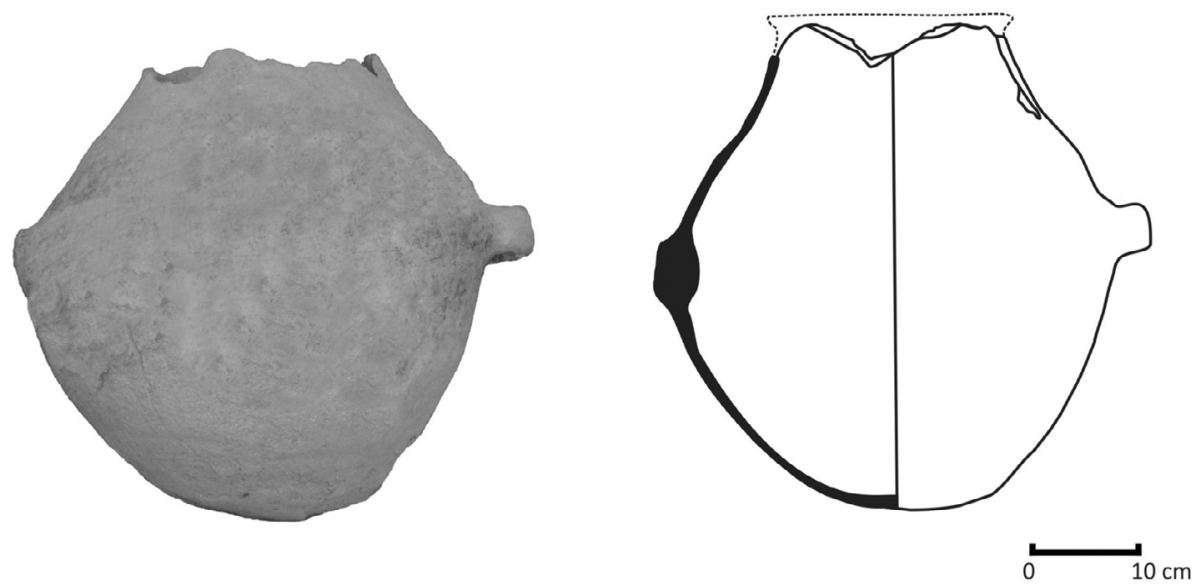

Figura 6: Olla ordinaria empleada como contenedor funerario en Soria 3.

(La fotografía ha sido retocada digitalmente).

de la parte media del cuerpo (Figura 6). La base, de contorno recto y $7 \mathrm{~cm}$ de diámetro, se encontraba muy erosionada, reduciendo la estabilidad de la vasija. La pieza fue modelada mediante la técnica de rodetes de arcilla y la superficie externa muestra un acabado alisado. Fue cocida en atmósfera oxidante y la pasta posee una alta porosidad, razón por la cual se desagrega fácilmente. En distintos sectores de la superficie externa del cuerpo se observan manchas negras que pueden responder a una fina capa de hollín o a manchas producto de la cocción. La pieza se encontró casi completa, con dos importantes grietas en el eje de las asas que la atraviesan desde el cuello hasta la base. Sus medidas aproximadas son: diámetro de boca $20 \mathrm{~cm}$ (estimado, debido a la fractura completa del borde), diámetro máximo $37 \mathrm{~cm}$ y altura $40 \mathrm{~cm}$.

La olla se encontraba dispuesta en el sedimento con las asas en un eje N-S, con una ligera inclinación hacia el este (ver Figura 5b). No se observó dentro de la cuadrícula ningún compartimento que cerrara el espacio de inhumación ni objetos que pudieran responder a un acompañamiento mortuorio.

La vasija contenía los restos de un subadulto. El esqueleto, al parecer articulado, debió estar sentado sobre el fondo de la vasija. El cráneo se encontró desplazado hacia el fondo, pero, por la disposición del cuerpo, se infiere que el individuo debió estar mirando al norte. A unos $25 \mathrm{~cm}$ de profundidad dentro de la olla se recuperaron dos piedras que pudieron ser las responsables de la fractura del borde y del desplazamiento y fractura del cráneo.

\subsection{Análisis bioarqueológico}

Se realizó un inventario siguiendo los criterios propuestos por Baker et al. (2005) y Scheuer y Black (2000). La observación se efectuó a ojo desnudo y las partes identificadas del individuo inhumado se registraron en un diagrama del esqueleto humano para subadultos tomado de Lewis (2007). La observación de las piezas dentales se 
Figura 7: Individuo de Soria 3: (a) diagrama del esqueleto con las partes óseas recuperadas (sombreadas); (b) fragmento de órbita ocular derecha con evidencias de puntillado poroso.

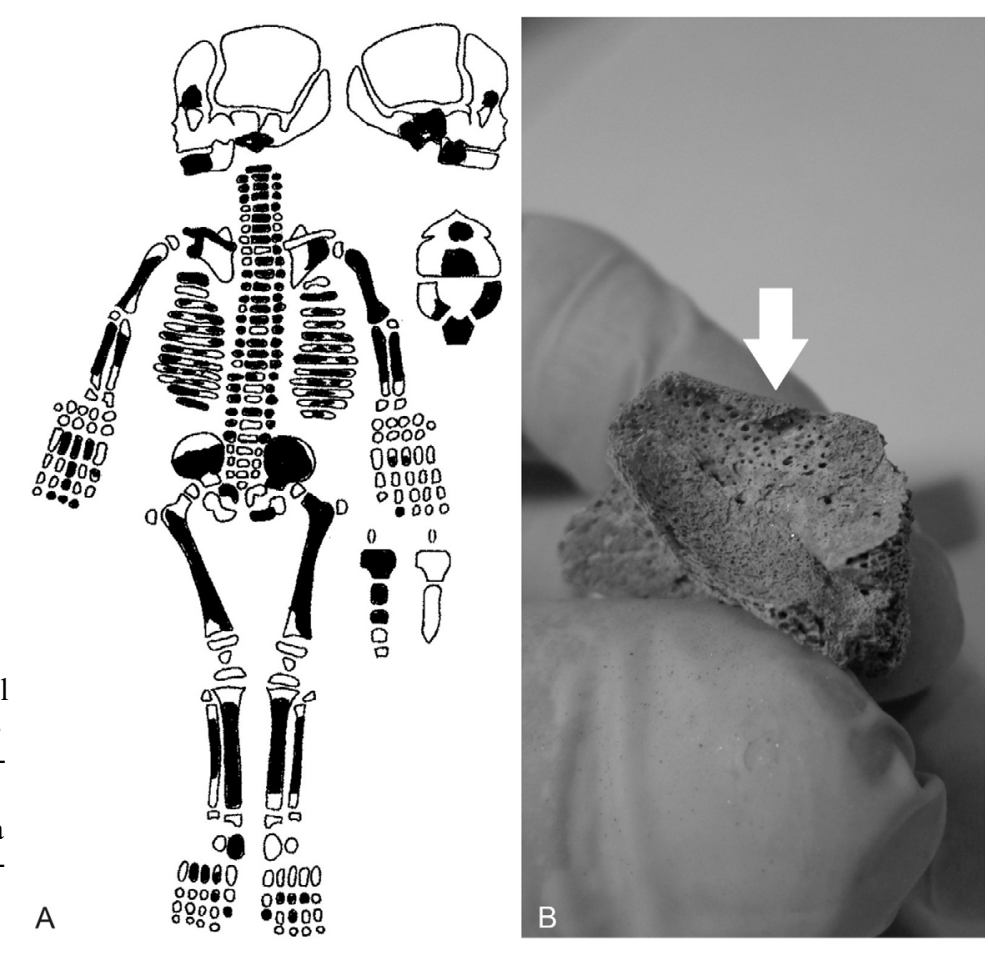

llevó a cabo con lupa binocular 10-40 X y se asentaron en los esquemas propuestos por Buikstra y Ubelaker (1994). La estimación de la edad esqueletal se realizó teniendo en cuenta el desarrollo dental (Buikstra y Ubelaker 1994) y las longitudes diafisarias de los huesos largos (Scheuer y Black 2000); seguimos luego la propuesta de Baldini y Baffi (2003) de utilizar las categorías etarias definidas por Bogin (1995) para grupos vivos. Con apoyo de lupa binocular se efectuó un examen macroscópico del esqueleto en busca de indicadores de procesos infecciosos y traumas e indicadores de estrés nutricional o fisiológico (Lewis 2007). Debido a que no existe un método ampliamente aceptado para la determinación de sexo en restos de subadultos, esta variable no se ha considerado.

El análisis de los restos óseos indica que su estado de preservación es regular, estando la mayor parte fragmentada y con los bordes erosionados. Del cráneo se recuperó cerca del $30 \%$ de los elementos; gran número de los fragmentos no pudo ser identificado con una parte esqueletal en particular. En su mayoría, éstos pertenecen a las partes más frágiles de la bóveda craneana, como los parietales y las porciones escamosas del temporal y del occipital. Es de destacar la presencia del yunque izquierdo y del martillo derecho; éstos, junto al estribo, son los huesos más pequeños del esqueleto y constituyen los osículos auditivos que se encuentran en el interior de la porción petrosa del hueso temporal. Los huesos pequeños de la cara -con excepción de las dos hemi-mandíbulas fragmentadas- están ausentes. De la dentición decidua se encontraron todos los dientes inferiores con excepción del canino izquierdo y de los superiores solamente el incisivo lateral derecho y los dos caninos. De la dentición 
definitiva, sólo un germen del primer molar. En dos dientes incisivos laterales -el inferior izquierdo y el superior derecho- se observa una ligera morfología «en pala», rasgo definido por Hrdlička (1920) y presente en las poblaciones amerindias (Turner 1989).

Con respecto al esqueleto post-craneal, está presente un 54\% de los elementos; la mayoría de las partes está muy desgastada, rota -como las costillas y las extremidades- o incompleta - como el caso de los omóplatos y los huesos de la cadera-. Los elementos más pequeños y frágiles, como los de las manos y de los pies, fueron los que menos se preservaron. En el diagrama de la Figura 7a se muestran sombreadas las partes óseas recuperadas.

De acuerdo con Buikstra y Ubelaker (1994), la edad dental corresponde a un neonato ( \pm 2 meses). Con base en la ecuación de regresión de edad, según la longitud máxima del húmero y del fémur izquierdos, se estimó una edad entre las 37 y 38 semanas de gestación $(36,92 \pm 2,33$ para el húmero y 38,35 $\pm 2,08$ para el fémur); en términos de Scheuer y Black (2000), se trata de un perinato o neonato a término. Teniendo en cuenta que un nacimiento a término ocurre entre las 37 y 42 semanas de gestación, este individuo no sobrevivió al parto a término o murió al poco tiempo de nacer, pudiendo en este caso haber sido amamantado por un corto período; por ello, siguiendo a Bogin (1995), se trataría de un infante.

El examen macroscópico reveló un puntillado poroso acotado en un fragmento muy pequeño de la órbita ocular derecha (Figura 7b), compatible con criba orbitalia, indicador no específico de anemia ferropénica (Lewis 2007). Esta lesión estaría indicando una situación de estrés fisiológico del feto en el útero, vinculado probablemente al estado de salud de la madre. Si bien no es posible afirmar que ésta haya sido la causa de muerte, bien podría haber coadyuvado a la misma (World Health Organization 2006).

\subsection{Otros hallazgos}

\subsubsection{Cerámica}

El material cerámico fue inspeccionado a ojo desnudo, contemplando para su clasificación características morfológicas, tecnológicas y de diseño. Pudieron recuperarse 285 fragmentos cerámicos, de los cuales 77 fueron excluidos del análisis estilístico por su tamaño inferior a los $2 \mathrm{~cm}$. La cerámica resultó ser un indicador contundente de cronología, y así, de los 208 fragmentos analizados, 200 corresponden a cerámica temprana. En este conjunto temprano destaca la cerámica Ordinaria $(n=164)$, de cocción en atmósfera oxidante, observándose un tratamiento de la superficie externa por alisado $(n=130)$, alisado con baño $(n=5)$, pulido $(n=16)$ e indeterminado $(n=13)$. Se registró asimismo alfarería Plomiza, obtenida en una atmósfera de cocción pobre en oxígeno $(n=29)$, en su mayoría con la superficie externa pulida y 2 ejemplares con decoración incisa.

Respecto a las formas generales de las vasijas, se reconocieron 23 fragmentos de formas abiertas, 71 de formas cerradas y 106 indeterminadas. Dentro del grupo 
de formas abiertas se pudo identificar la morfología de cuenco, representada por 20 fragmentos, de los cuales 17 eran de tipo Plomizo.

\subsubsection{Lítica}

El análisis tecnomorfológico y tipológico del conjunto lítico se realizó a ojo desnudo siguiendo los lineamientos propuestos por Aschero (1983). Los hallazgos líticos corresponden a medio molino, una mano de moler, un cuchillo de filo retocado confeccionado en filita, tres núcleos amorfos (sin diseño estandarizado) de andesita y 116 desechos de talla. Entre estos últimos, la materia prima predominante es la andesita $(\mathrm{n}=100)$, habiendo también lascas de cuarzo, cuarcita y rocas metamórficas, todas materias primas locales. Del total de desechos sólo 24 lascas se encuentran enteras.

Visto el conjunto lítico en forma integral -bajo porcentaje de núcleos y de lascas enteras, presencia de lascas pequeñas no corticales y alto porcentaje de lascas fracturadas con y sin talón-, el mismo estaría representando actividades vinculadas a la manufactura de herramientas (Sullivan y Rozen 1985).

\subsubsection{Fauna}

La identificación anatómica y taxonómica de los restos faunísticos se basó en la comparación morfológica con una colección osteológica de referencia siguiendo los parámetros definidos por Lyman (1994). La unidad de análisis fue el espécimen, entendido como todo fragmento o hueso completo. Para la cuantificación de los resultados utilizamos el Número de Especímenes (NSP), que incluye aquellos identificados y no identificados y el Número de Especímenes Identificados (NISP), en el que se incluyen sólo los huesos o fragmentos identificados al menos en el rango del orden taxonómico.

Se recuperaron 160 especímenes óseos, de los que se identificaron taxonómicamente 7 especímenes (4,3\% del conjunto), la mayoría de los cuales corresponde a Artiodactyla (NISP 3) o Camelidae (NISP 2).

\subsection{Cronología}

Las dataciones obtenidas fueron calibradas con la curva del Hemisferio sur ShCal13 (Hogg et al. 2013) y analizadas estadísticamente utilizando el programa OxCal v4.2 (Bronk Ramsey 2009).

Una primera datación por AMS de costillas del infante arrojó una fecha de $1659 \pm$ 46 AP (AA-99939). El rango de edad calibrada es de 340-573 d.C. $(p=95,4)$ (calibrado a $2 \sigma$ ). Una segunda datación sobre el húmero derecho arrojó una edad de $1575 \pm$ 25 AP (YU-2287) ${ }^{1}$, con un rango de edad calibrada de 438-592 d.C. $(\mathrm{p}=95,4)$. Debido a que los resultados de las muestras YU-2287 y AA-99939 pertenecen a un mismo

\footnotetext{
1 Muestras orgánicas para fechar el piso de ocupación de Soria 3 pudieron ser enviadas al laboratorio de la Universidad de Yamagata. Dado que ya contábamos con una datación del individuo inhumado realizado por el
} 
individuo, corresponde utilizar la fecha promediada de los dos. Para ello empleamos el test de homogeneidad T de Ward y Wilson (1978) que muestra que los valores son estadísticamente indistinguibles, pudiendo promediarse en $1594 \pm 22 \mathrm{AP}$, lo que da un rango de edad calibrada de $432-575$ d.C. $(p=95,4)$.

Una tercera datación se obtuvo de una muestra de carbón disperso proveniente del nivel 7 del R1, interpretado como parte del piso de ocupación de la unidad. Éste indicó una edad de $1675 \pm 20$ AP (YU-2136). El rango de edad calibrada es 366-517 d.C. $(p=95,4)$.

\section{Análisis de Soria 3-UA 11: un ejercicio interpretativo}

Todas las evidencias registradas en el sondeo de Soria 3-UA 11 indican que se trataría de una casa del período Temprano. En este sentido, Soria 2, en virtud de su proximidad y la riqueza de información que ha provisto, es el modelo ineludible para contrastar los hallazgos aquí mencionados. Los párrafos siguientes hacen un breve repaso de los distintos puntos abordados en el análisis que permiten ir bosquejando una modalidad de hábitat temprano en Andalhuala.

Ambos sitios comparten una definición difusa de su forma de planta en superficie. Soria 2 resultó de la apertura de un sondeo de $2 \mathrm{~m}^{2}$, efectuado en el ángulo que conformaban dos líneas cortas de piedras; sin embargo, extendida la excavación de la estructura, resultó que una de dichas líneas pertenecía al compartimiento de uno de los entierros de subadultos del sitio. El resto del trazado de los muros de los dos recintos de Soria 2 pudo ser descubierto solamente a partir de las excavaciones. De manera semejante, Soria 3 presenta por el momento un recinto de planta semicircular al cual se adosa un muro, no pudiendo determinarse aún si éste formó parte de un segundo recinto. En este sentido, ambas UA se encuentran emplazadas en el sector con mayor densidad arquitectónica del glacis producto de ocupaciones prolongadas en el tiempo que afectaron a sus plantas originales. A pesar de esto, se pudo corroborar por medio de las excavaciones que ambos sitios poseen una misma modalidad constructiva consistente en grandes mampuestos verticales en los muros, a manera de cimientos, escoltados por mampuestos más pequeños, con las caras planas dispuestas hacia el interior de las habitaciones, conformando muros de paños simples o dobles que definen habitaciones de planta subcuadrangular o subcircular. Estos rasgos constructivos compartidos configuran un estilo arquitectónico de tiempos tempranos en la MAB.

Existen asimismo claras semejanzas entre los materiales hallados en excavación en las superficies estables de ocupación, correspondientes a contextos domésticos. Por un lado, los mismos estilos alfareros fueron empleados en las dos antiguas viviendas. Dentro del conjunto ordinario recuperado en Soria 3-UA 11, la alta proporción de fragmentos de cocción en atmósfera oxidante pertenecientes a formas restringidas es coherente con lo analizado en Soria 2, siendo las formas restringidas en su mayoría ollas globulares (Baigorria Di Scala 2009). Dentro del conjunto fino, sobresale la presencia de

laboratorio de la Universidad de Arizona, se optó por replicar esta datación para tener una muestra de control entre los valores de ambos laboratorios. 
fragmentos plomizos de cocción pobre en oxígeno y superficies pulidas, la mayor parte correspondientes a cuencos (Spano 2011). Por su parte, el material lítico presenta las mismas características que las registradas en Soria 2 (Carbonelli 2011). Ambos conjuntos líticos parecen ser el resultado de eventos de manufactura de instrumentos con baja inversión de esfuerzo tecnológico en la talla. En lo referente al conjunto óseo, si bien es pequeño como para hacer mayores inferencias, la presencia de huesos de Camelidae y Artyodactila es congruente con lo observado en Soria 2 (Belotti 2011).

Respecto al entierro del infante, la olla de tipo ordinario que servía de contenedor del individuo inhumado en Soria 3-UA 11 presenta características morfológicas, técnicas y decorativas que la emparentan con las tres ollas recuperadas en los entierros de Soria 2 (Figura 8). Comparte su forma globular, su generoso cuerpo, su boca estrecha, su pasta gruesa, la técnica de elaboración mediante rollos, la cocción en atmósfera oxidante y el acabado de superficie por alisado. Sin embargo, la pieza de Soria 3 exhibe algunos elementos que la distinguen sutilmente: posee dos asas horizontales, a diferencia de las ollas completas de Soria 2, que sólo poseían una en posición oblicua; la factura general de la pieza parece más tosca, en cuanto al alisado de la superficie externa, no tan prolijo como las de Soria 2, y a la porosidad de la pasta, que la torna disgregable (a diferencia de las ollas de Soria 2, de pasta mucho más compacta); y la forma conseguida por el alfarero no alcanza la simetría que poseen las demás. La modalidad de entierro también se comparte: hasta el momento, los tres entierros primarios registrados en Andalhuala Banda corresponden a infantes contenidos en ollas de tipo ordinario cuyas características estilísticas son asimismo indistinguibles de aquellas empleadas en contextos domésticos. En este sentido, no parece haber habido una cualidad objetiva que operara como diacrítico de las vasijas que debían ser usadas como contenedores funerarios; más bien, cotidianidad y eventos especiales no se encontraban escindidos y la alfarería objetivó los lazos que unían vida y muerte.

En cuanto a los individuos inhumados, la estimación de la edad de los dos infantes de Soria 2 y el de Soria 3 permitió establecer que se trata de perinatos o neonatos a término. A partir del examen macroscópico y radiológico - este último sólo para los individuos de Soria 2- no se pudo determinar en ningún caso la causa de muerte; sin embargo, los análisis efectuados a los perinatos indicarían que éstos no sobrevivieron al parto a término, o que murieron inmediatamente después de nacer o a los pocos días. En uno de los individuos de Soria 2 se detectó puntillado poroso en un sector de la bóveda craneana, compatible con la hiperostosis porótica, lesión probablemente vinculada con una anemia resultante de deficiencia de hierro (Lewis 2007); una patología similar (criba orbitalia) se observó también en el perinato de Soria 3, lo que lleva a pensar en la posibilidad de que estas situaciones de estrés fisiológico sufridas por los fetos en el útero y vinculadas al estado de salud materno, pudieron enmarcarse en procesos mayores a nivel de la población temprana de la MAB, aunque es claro que las muestras no son por el momento suficientes para tratar esta problemática.

Por último, el refinado estudio de la sucesión estratigráfica de Soria 2 permitió distinguir entre el uso de la estructura como espacio de vivienda y la posterior utilización para la ejecución de rituales funerarios ${ }^{2}$. Por otro lado, la ausencia de cortes en

2 Para una descripción detallada de la secuencia estratigráfica remitimos a Spano et al. 2014. 


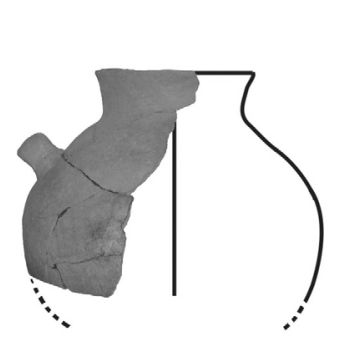

a

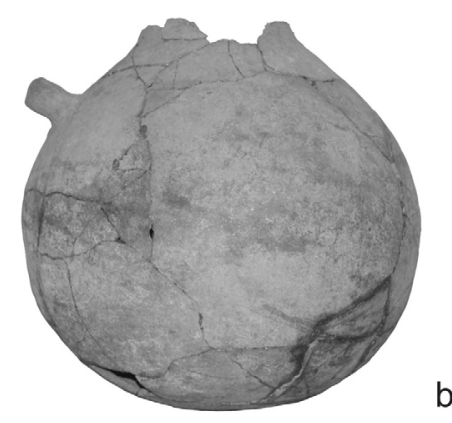

b

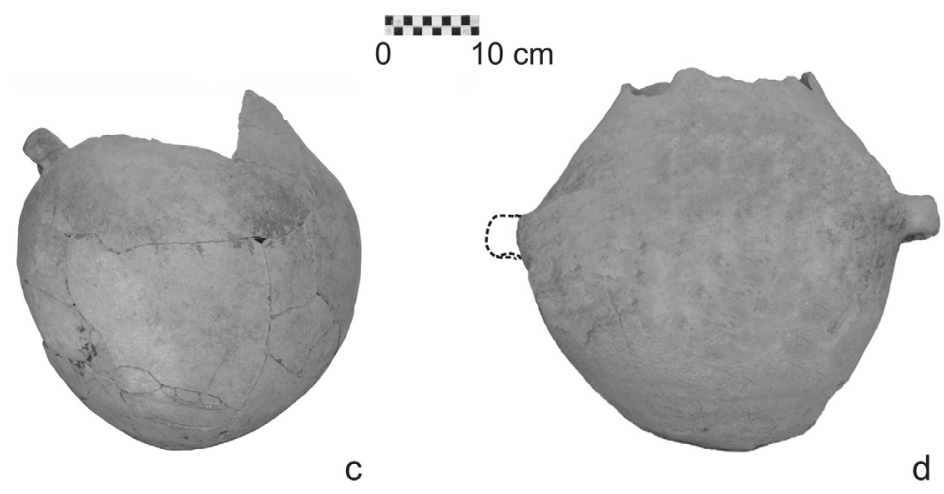

Figura 8: Ollas-urnas de Soria 2 (a, b y c) y Soria 3 (d).

el relleno depositado por encima del piso de ocupación que indicaran la extracción de sedimento para generar los pozos destinados a las inhumaciones, permitió inferir que la acumulación del relleno fue posterior a los entierros y que éstos pudieron constituir un evento seguido al abandono de la vivienda (Spano et al. 2014: 160). De manera análoga, la secuencia estratigráfica de Soria 3 ha permitido determinar que el ingreso del entierro fue un evento ocurrido con posterioridad a la conformación y uso del estrato que conformaba el piso de ocupación, pero mediando un lapso corto de tiempo debido a la ausencia de cortes en el relleno de la unidad arquitectónica. En este sentido, las edades radiocarbónicas obtenidas en ambos sitios colocan en un mismo bloque cronológico las historias de dos casas, sus tiempos sociales de vida, muerte y abandono. Asimismo, presentan el mismo patrón de pisos ocupacionales ligeramente más tempranos que los entierros, reforzando las interpretaciones estratigráficas. Si bien se trata aún de información acotada, considerando la extensión espacial de la MAB, la consistencia de las dataciones fortalece el planteamiento de la existencia de un modo de hacer local que pudo haber formado parte de una cosmovisión compartida a escala vallista.

Las evidencias, si bien preliminares, apuntan a la existencia de un sistema de asentamiento en el cual las unidades domésticas se dispersan en terrenos propicios para la actividad agropastoril. En este sentido, la ocupación temprana en Andalhuala sería semejante a otros asentamientos tempranos del Noroeste argentino como La Bolsa 1, Bajo Los Cardones y Loma Alta, entre otros (Gazi 2012; Pastor y Rivero 2004; Scattolin 1990), instalados en terrazas de pendiente moderada, buenos suelos, inso- 
lación para los cultivos y accesibilidad al agua de riego. Se trataría de comunidades pequeñas, con un bajo nivel de diferenciación social (posiblemente regidas por distinciones sexuales, grupos de edad y/o pertenencia a linajes), en un paisaje social que carecía de marcados contrastes, para el cual cabe suponer que las relaciones interpersonales y la apropiación de recursos se encontraban reguladas por los derechos y obligaciones inherentes a los vínculos de parentesco (Núñez 1978).

El desequilibrio ya mencionado entre las dimensiones de las áreas excavadas de los dos sitios - consistente con los objetivos de investigación perseguidos en cada casonos impone cierta cautela a la hora de realizar comparaciones de sintonía más fina entre los dos contextos en tanto que ámbitos pretéritos de cotidianidad. De todos modos, resulta claro que Soria 2 y Soria 3 comparten tanto estilos arquitectónicos como artefactuales, elemento que nos alienta a pensar que las personas que habitaron en el pasado estos espacios formaron parte de un mismo entramado de prácticas, experiencias y percepciones sobre el mundo, es decir, de un mismo colectivo social. Por consiguiente, la afinidad de estilo alfarero y arquitectónico se torna, aquí, significativa para tender puentes hacia una interpretación de ciertas prácticas locales en el pasado.

\section{Materialidad temprana y ritual fúnebre, I: las ollas ordinarias como urnas funerarias}

Los procesos involucrados en la producción de cultura material componen una amalgama de decisiones, elecciones, necesidades y voluntades, tanto individuales como colectivas. Tal es el caso de las manufacturas alfarera y arquitectónica, en las cuales la morfología, la tecnología y el diseño, integrados, constituyen el estilo en tanto modo de hacer, contextualizado social e históricamente; el estilo es a su vez un rasgo observable que posibilita reconocer a un objeto o eventualmente a una manifestación arquitectónica como parte de un conjunto vinculado a determinado espacio y tiempo y a ciertas personas (Hodder 1990; Miller 1985).

La olla que contenía los restos del neonato en Soria 3 se vincula estilísticamente con aquellas asociadas a los entierros de Soria 2. Si observamos en conjunto las cuatro ollas, percibimos un aire de familia que indica su pertenencia a un modo de hacer compartido en Andalhuala Banda en los primeros siglos de la era cristiana. A su vez, las vasijas usadas en todos los entierros registrados pertenecen a la misma clase de alfarería ordinaria utilizada en las prácticas cotidianas, cuando las viviendas se encontraban plenas de actividad humana; esta alfarería es reconocible en los restos recuperados del piso de ocupación de Soria 2 y Soria 3. En la vida diaria, esta clase de ollas, debido al diámetro pequeño de boca y a su alta capacidad potencial, eran adecuadas para contener cantidades importantes de líquidos, ya que las aberturas reducidas ayudan a evitar el derrame y la evaporación de los fluidos. De modo similar y desde una perspectiva funcional, en comparación con otros soportes materiales que las poblaciones tempranas tenían disponibles, estos recipientes de cerámica resultaban apropiados para la protección del cuerpo del difunto depositado en su interior, aislando el contenido con respecto a la matriz sedimentaria y manteniendo a los restos humanos acotados espacialmente dentro de las paredes de la olla (Alvarado 
1997). Por otro lado, como plantean Ortega y Cervantes (2009), el empleo de ollas globulares como urnas resulta una metáfora de regreso al vientre materno. En este sentido es sugerente que el uso de las ollas se reservara usualmente a fetos o infantes que no sobrevivieron el parto o fallecieron al poco tiempo de nacer, habiendo sido el útero su principal morada en la gestación.

Las semejanzas estilísticas entre ollas funerarias y domésticas pueden deberse a que se mantuvieron las mismas pautas en la elaboración de cerámica a lo largo del período Temprano; producida la muerte de algún infante de la comunidad, se reciclaron ciertos recipientes del equipamiento doméstico, resignificándolos como contenedores apropiados para los restos. $\mathrm{O}$, tal vez, las personas que enterraron a sus muertos recurrieron a vasijas de otros tiempos, atesoradas durante generaciones y valoradas de manera significativa, como vehículos, y a la vez recipientes, de la memoria grupal. Alternativamente, pudieron confeccionarse estas ollas específicamente para su uso funerario; tal situación explicaría la hechura rústica de la pieza de Soria 3, elaborada con premura ante el fallecimiento inesperado del bebé, tomando como modelo los contenedores que se usaban en las tareas domésticas (aunque esta hipótesis no podría aplicarse a las otras tres ollas).

Cualquiera que sea el caso, estamos hablando de piezas que pertenecen a un mismo conjunto alfarero; si las tuviéramos descontextualizadas no encontraríamos mayores diferencias entre ellas. Un elemento en común es su condición de contenedores; el hecho de la muerte cambiaría, entonces, la significación primaria de estos objetos, transfiriéndolos a otra clase de uso -ahora funerario- y manteniendo, no obstante, la funcionalidad al constituirse como espacio receptivo.

\section{Materialidad temprana y ritual fúnebre, II: las casas como tumbas}

$\mathrm{Al}$ igual que las vasijas, las casas también se erigen como espacio receptivo en la muerte: tras su abandono como lugar de residencia (inmediatamente después o en algún momento dentro de un lapso de alrededor de 200 años), las mismas fueron utilizadas como espacios funerarios por personas que habrían compartido con los antiguos habitantes de las viviendas ciertas pautas culturales, tal como venimos discutiendo líneas arriba. La datación obtenida del piso de ocupación de Soria 3 es algo más antigua que el entierro. A su vez, todas las dataciones son ligeramente más tardías que las de las inhumaciones de Soria 2. Esto sugiere la existencia de prácticas de desocupación de las casas y habitación y/o construcción de otras por parte de una misma comunidad dentro del ámbito de la aldea.

Los motivos que llevaron a la desocupación de las casas permanecen inciertos. Tal vez los habitantes de la casa optaron por residir en otra vivienda, y efectuaron los entierros seguidamente a la desocupación o tiempo después, como una suerte de clausura del hogar, ahora transformado en lugar de reposo final de los infantes muertos. No obstante, de acuerdo con el planteamiento de Nelson (2000), si bien los abandonos definitivos suelen requerir cierres rituales -por ejemplo incendios-, los movimientos locales generalmente no los necesitan, dado que los sitios continúan siendo visitados u observados. Creemos que este último escenario representa el caso de las unida- 
des domésticas tempranas de la $\mathrm{MAB}$, desocupadas -sin evidencias de incendios de clausura- y luego reutilizadas como ámbito funerario, donde esos antiguos lugares residenciales continuaron siendo parte de la vida cotidiana de las personas, ya no como ámbito de reproducción doméstica, sino como un espacio de conmemoración y protección de los pequeños difuntos.

En un modo de vida en el cual las relaciones interpersonales y los lazos con la tierra se encontraban regulados por los vínculos de parentesco, cobra especial relevancia la reutilización de las antiguas unidades domésticas como lugar para el entierro de los infantes de la comunidad aldeana. Las prácticas funerarias registradas para las sociedades tempranas del Noroeste argentino, incluyen dos modalidades de inhumación: la presencia de cámaras de piedra debajo del piso de los patios de manera contemporánea al uso doméstico (a veces sobresaliendo los accesos por encima del piso) y la existencia de pequeñas necrópolis separadas de los sectores residenciales y de producción (Raffino 2007: 230-232). En este sentido, las ocupaciones tempranas de la $\mathrm{MAB}$ evidencian una modalidad de práctica funeraria distinta y poco conocida: el uso de casas deshabitadas para el entierro de infantes. Esta práctica funeraria refuerza la idea, mencionada líneas arriba, de la existencia de un patrón de asentamiento con una movilidad a escala local, en el cual el ritual mortuorio resulta un indicador relevante al dar cuenta de que esas casas no fueron del todo abandonadas, sino que siguieron formando parte del espacio habitado.

\section{Comentarios finales}

El objetivo de este trabajo ha sido aportar información novedosa sobre una problemática de larga data relacionada con el carácter que tuvieron las primeras ocupaciones agroalfareras de Yocavil. En este sentido, Soria 2 se ha constituido como un caso «testigo» de la ocupación temprana en Andalhuala con el cual contrastar las evidencias recuperadas en Soria 3, permitiéndonos realizar un ejercicio interpretativo de este acotado contexto arqueológico. La correspondencia entre ambos lugares en términos de materialidad doméstica y funeraria posibilita, más allá del palimpsesto de ocupaciones que es hoy en día la Mesada de Andalhuala Banda, avanzar en la configuración de un patrón local de uso de este paisaje temprano relacionado con prácticas tanto ordinarias como extraordinarias.

En Soria 3 una parte de lo habitual y lo ritual se inscribió en los mismos contextos materiales, expresando los límites difusos entre vida y muerte que pudieron existir en tiempos pasados en estas comunidades: el empleo de la vasija ordinaria, otrora usada para almacenar, como contenedor apropiado para el difunto y tal vez como metáfora del regreso al vientre materno, la elección de la casa como afirmación de su pertenencia al grupo con el cual no pudo interactuar en vida. En definitiva, vida cotidiana y muerte se intersectan en el cuerpo de las ollas y en las antiguas viviendas, las cuales enlazan a través de su materialidad distintos tiempos sociales.

Agradecimientos: A Juan Pablo Carbonelli, Carlos Belotti, Catriel Greco y Jason Nesbitt por su colaboración en distintas instancias del análisis. 


\section{Referencias bibliográficas}

Alvarado, Margarita

1997 «La tradición de los grandes cántaros: reflexiones para una estética del 'envase'». Aisthesis 30: 105-123.

Álvarez Larrain, Alina

2015 Habitar una región. Espacialidad arquitectónica y construcción de paisajes en Andalhuala, valle de Yocavil (Catamarca, Argentina). Tesis Doctoral inédita. Facultad de Filosofía y Letras, Universidad de Buenos Aires, Buenos Aires.

Álvarez Larrain, Alina y Sonia Lanzelotti

2013 «Habitar y cultivar en el este del valle de Yocavil», en La Espacialidad en Arqueología. Enfoques, métodos y aplicaciones, Inés Gordillo y José M. Vaquer, ed., pp. 151-190. Quito: Editorial Abya-Yala.

AsCHERo, Carlos

1983 «Ensayo para una clasificación morfológica de artefactos líticos». Manuscrito inédito para la Cátedra de Ergología y Tecnología. Facultad de Filosofía y Letras, Universidad de Buenos Aires, Buenos Aires.

BAIGORRIA Di Scala, Jennifer

2009 El sitio Formativo Soria 2: Estudio tecno-morfológico del conjunto cerámico ordinario. Tesis de Licenciatura inédita, Facultad de Filosofía y Letras, Universidad de Buenos Aires, Buenos Aires.

BAILEY, Geoff

2007 «Time Perspectives, Palimpsests and the Archaeology of Time». Journal of Anthropological Archaeology 26 (2): 198-223.

BAKer, Brenda J., Tosha L. Dupras y Matthew W. TocherI

2005 The Osteology of Infants and Children. College Station: Texas A\&M University Press.

BALDINI, Lidia e Inés BAFFI

2003 «Niños en vasijas. Entierros tardíos del valle Calchaquí. (Salta)». Runa 24 (1): 43-62.

Belotti, Carlos

2011 «Zooarqueología del sitio formativo Soria 2, valle de Yocavil (Catamarca), siglo I d.C.». Revista del Museo de Antropología 4: 3-16.

Bogin, Barry

1995 "Growth and Development: Recent Evolutionary and Biocultural Research», en Biological Anthropology. The State of the Science, Noel T. Boaz y Linda D. Wolfe, ed., pp. 49-70. Oregon: International Institute for Human Evolutionary Research.

Bronk RamSEY, Christopher

2009 «Bayesian Analysis of Radiocarbon Dates». Radiocarbon 51 (1): 337-360.

Buikstra, Jane E. y Douglas H. UBELAKER

1994 Standards for Data Collection from Human Skeletal Remains. Arkansas: Arkansas Archaeological Survey Research Series No. 44.

CArbonelli, Juan Pablo

2011 «'Motivos porque y para’ en la tecnología lítica de un sitio formativo en el Valle de Yocavil, provincia de Catamarca». Intersecciones en Antropología 12: 31-45. 
GAZI, Verónica

2012 «Prácticas cotidianas y reproducción social. Un estudio de los ámbitos residenciales del primer milenio D.C. en el Valle de Tafí». Comechingonia Virtual 6 (1): 134-151.

GonZÁLEZ, Alberto Rex y Víctor NúÑEZ REGUEIRO

1960 «Preliminary Report on Archaeological Research in Tafí del Valle, N. W. Argentine». Akten der 34 Internationalen Amerikanisten Kongress, pp. 485-496.

Greco, Catriel

2012 Integración de datos arqueológicos, radiocarbónicos y geofísicos para la construcción de una cronología de Yocavil y alrededores. Tesis Doctoral inédita. Facultad de Filosofía y Letras, Universidad de Buenos Aires, Buenos Aires.

HODDER, Ian

1990 «Style as Historical Quality», en The Uses of Style in Archaeology, Margaret W. Conkey y Christine A. Hastorf, ed., pp. 44-51. Cambridge: Cambridge University Press.

Hogg, Alan G., Quan Hua, Paul G. Blackwell, Mu Niu, Caitlin E. Buck, Thomas P. Guilderson, Timothy J. Heaton, Jonathan G. Palmer, Paula J. Reimer, Ron W. Reimer, Christian S. TURNeY, Susan R. ZIMMERMAN

2013 «SHCal13 Southern Hemisphere Calibration, 0-50,000 Years cal BP». Radiocarbon 55 (4): 1889-1903.

HRDLIČKA, Aleš

1920 «Shovel-Shaped Teeth». American Journal of Physical Anthropology 3 (4): 429465.

LEwIS, Mary E.

2007 The Bioarchaeology of Children: Perspectives from Biological and Forensic Anthropology. Cambridge: Cambridge University Press.

LYMAN, R. Lee

1994 Vertebrate Taphonomy. Cambridge: Cambridge University Press.

Maldonado, Marío G., Álvaro J. Cordomí, Liliana Neder y María M. SAmpietro Vattuone

2012 «Tiempo y espacio: el sitio «Talapazo» (Valle de Yocavil, Provincia de Tucumán)». La Zaranda de Ideas 8 (2): 101-117.

Miller, Daniel

1985 Artefacts as Categories. A Study of Ceramic Variability in Central India. Cambridge: Cambridge University Press.

NeLson, Margaret C.

2000 «Abandonment: Conceptualization, Representation, and Social Change», en Social Theory in Archaeology, Michael Schiffer, ed., pp. 52-62. Salt Lake City: University of Utah Press.

NúÑEZ REGUEIRO, Víctor

1978 «Considerations on the Periodizations of Northwest Argentina», en Advances in Andean Archaeology, David Browman, ed., pp. 453-484. París: Mouton.

OLIVIER, Laurent

1999 «The Hochdorf 'Princely' Grave and the Question of the Nature of Archaeological Funerary Assemblages», en Time and Archaeology, Tim Murray, ed., pp. 109-138. Londres: Routledge. 
Ortega Palma, Albertina y Jorge Cervantes Martínez

2009 «Cuerpos inhumados en vasijas del Estado de Campeche». Estudios de Cultura Maya 36: 67-86.

Palamarczuk, Valeria, Romina Spano, Daniel Magnífico, Florencia Weber, Soledad LóPez y Mariano MANASIEWICZ

2007 «Soria 2. Apuntes sobre un sitio temprano en el valle de Yocavil (Catamarca, Argentina)». Intersecciones en Antropología 8: 121-134.

Pastor, Sebastián y Diego Rivero

2004 «Nuevas evidencias en torno a la ocupación agroalfarera temprana del valle de Yocavil», en Mosaico. Trabajos en antropología social y arqueología, Mariana Carballido Calatayud, ed., pp. 189-199. Buenos Aires: INAPL.

RAFFINO, Rodolfo

2007 Poblaciones indígenas de la Argentina. Buenos Aires: Editorial TEA.

ScatTolin, María Cristina

1990 «Dos asentamientos formativos al pie del Aconquija: el sitio Loma Alta». Gaceta Arqueológica Andina 5 (17): 85-100.

2000 «Santa María durante el Primer Milenio A.D. ¿Tierra baldía?». Etnografiska Museet $i$ Goteborg. Arstryck 1995-1998: 63-83.

2007 «Santa María antes del año mil. Fechas y materiales para una historia cultural», en Sociedades Precolombinas Surandinas. Temporalidad, Interacción y Dinámica cultural del NOA en el ámbito de los Andes Centro-Sur, Verónica Williams, Beatriz Ventura, Adriana Callegari y Hugo Yacobaccio, eds., pp. 203-219. Buenos Aires: Edición de los autores.

2010 «La organización del hábitat precalchaquí (500 a.C.-1000 d.C.)», en El hábitat prehispánico. Arqueología de la arquitectura y de la construcción del espacio organizado, $\mathrm{M}^{\mathrm{a}}$ Ester Albeck, $\mathrm{M}^{\mathrm{a}}$ Cristina Scattolin y Alejandra Korstanje, eds., pp. 13-51. San Salvador de Jujuy: FHyCS, UNJu.

ScheUer, Louise y Sue M. Black

2000 Developmental Juvenile Osteology. Londres: Academic Press.

SPANO, Romina

2011 «Primera sistematización de las características estilísticas de la alfarería fina del sitio Soria 2 (Valle de Yocavil, Noroeste argentino)». Revista del Museo de Antropología 4: 127-144.

Spano, Romina, M. Solange Grimoldi y Valeria PalamarczuK

2014 «Morir temprano. Entierros de infantes en un espacio doméstico formativo de Yocavil, Noroeste Argentino». Estudios. Antropología-Historia (Nueva Serie) 2: 141-173.

Sullivan, Alan P. y Kenneth C. Rozen

1985 «Debitage, Analysis and Archaeological Interpretation». American Antiquity 50 (4): 755-779.

TARragó, Myriam N. y María Cristina Scattolin

1999 «La problemática del Período Formativo en el valle de Santa María». Actas del XII Congreso Nacional de Arqueología Argentina I: 142-153. La Plata.

TURNER, Christy G.

1989 «Teeth and Prehistory in Asia». Scientific American 260: 88-96. 
WARD, Graeme K. y Sue R. WILSON

1978 «Procedures for Combining Radiocarbon Age Determinations: A Critique». Archaeometry 20 (1): 19-31.

World Health ORganization

2006 Neonatal and Perinatal Mortality. Country, Regional and Global Estimates. Ginebra: World Health Organization. 